\title{
580.
}

\section{ON THE NUMBER OF DISTINCT TERMS IN A SYMMETRICAL OR PARTIALLY SYMMETRICAL DETERMINANT.}

[From the Monthly Notices of the Royal Astronomical Society, vol. xxxiv. (1873-1874), pp. 303-307, and p. 335.]

THE determination of a set of unknown quantities by the method of least squares is effected by means of formulæ depending on symmetrical or partially symmetrical determinants; and it is interesting to have an expression for the number of distinct terms in such a determinant.

The terms of a determinant are represented as duads, and the determinant itself as a bicolumn; viz. we write, for instance,

$$
\left\{\begin{array}{l}
a a \\
b b \\
p p^{\prime} \\
q q^{\prime}
\end{array}\right\} \text { to represent the determinants }\left|\begin{array}{cccc}
a a, & a b, & a p^{\prime}, & a q^{\prime} \\
b a, & b b, & b p^{\prime}, & b q^{\prime} \\
p a, & p b, & p p^{\prime}, & p q^{\prime} \\
q a, & q b, & q p^{\prime}, & q q^{\prime}
\end{array}\right| .
$$

This being so if the duads are such that in general $r s=s r$, then the determinant is wholly or partially symmetrical; viz. the determinant just written down, for which the bicolumn contains such symbols as $p p^{\prime}$ and $q q^{\prime}$, (each letter $p, q, \ldots$ being distinct from every letter $\left.p^{\prime}, q^{\prime}, \ldots\right)$ is partially symmetrical, but a determinant such as $\left\{\begin{array}{l}a a \\ b b \\ c c\end{array}\right)$ is wholly symmetrical. A determinant for which the bicolumn has $m$ rows $a a, b b$, \&c., and $n$ rows $p p^{\prime}, q q^{\prime}$, \&c. is called a determinant $(m, n)$; and the number of distinct terms in the developed expression of the determinant is taken to be $\phi(m, n)$; the problem is to find the number of distinct terms $\phi(m, n)$.

c. IX. 
Consider a determinant $(m, n)$ where $n$ is not $=0$; for instance, the determinant above written down, which is $(2,2)$; this contains terms multiplied by $q a, q b, q p^{\prime}, q q^{\prime}$ respectively: where, disregarding signs, the whole factor multiplied by $q a$ is $\left\{\begin{array}{c}b b \\ a p^{\prime} \\ p q^{\prime}\end{array}\right\}$, which is a determinant $(1,2)$, and similarly the whole factor multiplied by $q b$ is a determinant $(1,2)$. But the whole factor multiplied by $q p^{\prime}$ is the determinant $\left\{\begin{array}{c}a a \\ b b \\ p q^{\prime}\end{array}\right\}$, which is a determinant $(2,1)$, and the whole factor multiplied by $q q^{\prime}$ is also a determinant $(2,1)$.

Hence, observing that $q a, q b, q p^{\prime}, q q^{\prime}$ are distinct terms occurring only in the last line of the determinant, the number of distinct terms is equal to the sum of the numbers of distinct terms in the several component parts, or we have

and so in general :

$$
\phi(2,2)=2 \phi(1,2)+2 \phi(2,1)
$$

$$
\phi(m, n)=m \phi(m-1, n)+n \phi(m, n-1) .
$$

Consider next a completely symmetrical determinant $(m, 0)$; for instance $(4,0)$, the determinant

$$
\left\{\begin{array}{l}
a a \\
b b \\
c c \\
d d
\end{array}\right\},=\left|\begin{array}{cccc}
a a, & a b, & a c, & a d \\
b a, & b b, & b c, & b d \\
c a, & c b, & c c, & c d \\
d a, & d b, & d c, & d d
\end{array}\right| .
$$

We have first the terms containing $d d$; the whole factor is $\left\{\begin{array}{l}a a \\ b b \\ c c\end{array}\right\}$, which is a determinant $(3,0)$; secondly, the terms containing $a d . d a$, or the like combinations, $b d . d b$ or $c d . d c$; the whole factor multiplied by $a d . d a$ is $\left\{\begin{array}{l}a a \\ b b\end{array}\right\}$, which is a determinant $(2,0)$; thirdly, the terms containing $a d . d b+b d . d a,=2 a d . b d$; or the like combinations $2 a d . c d$ or $2 b d . c d$ : the whole factor multiplying the term $2 a d . b d$ is $\left\{\begin{array}{l}c c \\ b a\end{array}\right\}$, which is a determinant $(1,1)$. Hence observing that $a d, b d, c d,=d a, d b, d c$, and $d d$ are terms occurring only in the last line and column of the original determinant, it is clear that the number of distinct terms in the original determinant is equal to the sum of the numbers of distinct terms in the component parts, or that we have $\phi(4,0)=\phi(3,0)+3 \phi(2,0)+3 \phi(1,1) ;$ and so in general :

$$
\phi(m, 0)=\phi(m-1,0)+m \phi(m-2,0)+\frac{m \cdot m-1}{2} \phi(m-3,1) .
$$


The two equations of differences, together with the initial values $\phi(0,0)=1$, $\phi(1,0)=\phi(0,1)=1, \phi(2,0)=\phi(1,1)=\phi(1,2)=2$, enable the calculation of the successive values of $\phi(m, n)$ : viz. arranging these in the order

$$
\begin{gathered}
\phi(0,0), \\
\phi(1,0), \quad \phi(0,1), \\
\phi(2,0), \quad \phi(1,1), \quad \phi(0,2), \\
\phi(3,0), \& \text { c., \&c., }
\end{gathered}
$$

we calculate simultaneously the lines $\phi(m, 0), \phi(m, 1)$; and thence successively the remaining lines $\phi(m, 2), \phi(m, 3)$, \&c. : the values up to $m+n=6$ being in fact

1 ,

$$
\begin{array}{ll}
1, & 1, \\
2, & 2, \quad 2,
\end{array}
$$

$5,6,6,6$,

$17,23,24,24,24$,

$73,109,118,120,120,120$,

$388, \quad 618,690,714,720,720,720$ :

where the process for the first two lines is

$$
\begin{aligned}
& 5=2+2.1+.1, \quad 6=2.2+2, \\
& 17=5+3 \cdot 2+3 \cdot 2, \quad 23=3 \cdot 6+5 \text {, } \\
& 73=17+{ }_{4} .5+6.6, \quad 109={ }_{4} .23+17 \text {, } \\
& 388=73+5.17+.23, \quad 618={ }_{5} .109+23 \text {, }
\end{aligned}
$$

the larger figures being those of the two lines, and the smaller ones numerical multipliers. And then for the third line, fourth line, \&c., we have

and so on.

$$
\begin{array}{rlrl}
6 & =\text { r. } 2+2 . \quad 2, & 120=2 \cdot 24+3 \cdot 24, \\
24={ }_{2} . \quad 6+2 . \quad 6, & 714=3 \cdot 120+{ }_{4} \cdot 118, \\
118 & ={ }_{3} \cdot 24+2.23, & : \\
690 & ={ }_{4} \cdot 118+2.109, &
\end{array}
$$

This is, in fact, the easiest way of obtaining the actual numerical values; but we may obtain an analytical formula. Considering the two equations

$$
\begin{gathered}
\phi(m, 1)=m \phi(m-1,1)+\phi(m, 0), \\
\phi(m, 0)=\phi(m-1,0)+m \phi(m-2,0)+\frac{m \cdot m-1}{2} \phi(m-3,1) ;
\end{gathered}
$$


and using the first of these to eliminate the term $\phi(m-3,1)$ and resulting terms $\phi(m-4,1)$, \&c. which present themselves in the second equation, this, after a succession of reductions, becomes

$$
\begin{aligned}
& \phi(m, 0)= \phi(m-1,0) \\
&+(m-1) \phi(m-2,0) \\
&+\frac{m . m-1}{2}\{\phi(m-3,0) \\
&+(m-3) \phi(m-4,0) \\
& \\
&+(m-3) \ldots 3.2 \phi(1,0) \\
&+(m-3) \ldots 3.2 .1\} \\
&+
\end{aligned}
$$

or, observing that the last term $(m-3) \ldots 3.2 .1$ is, in fact, $=(m-3) \ldots 3.2 .1 \phi(0,0)$, this may be written:

$$
\begin{array}{r}
2 \phi(m, 0)-\phi(m-1,0)-(m-1) \phi(m-2,0)= \\
\phi(m-1,0) \\
+(m-1) \phi(m-2,0) \\
+(m-1)(m-2) \phi(m-3,0) \\
\vdots \\
+(m-1) \ldots 3.2 .1 \phi(\quad 0,0) .
\end{array}
$$

And hence assuming

$$
u=\phi(0,0)+\frac{x}{1} \phi(1,0)+\frac{x^{2}}{1.2} \phi(2,0)+\ldots+\frac{x^{m}}{1.2 \ldots m} \phi(m, 0)+\ldots
$$

we find at once

$$
2 \frac{d u}{d x}-u-x u=\frac{u}{1-x}
$$

that is,

$$
2 \frac{d u}{u}=d x\left(1+x+\frac{1}{1-x}\right)
$$

or integrating and determining the constant so that $u$ shall become $=1$ for $x=0$, we have

$$
u=\frac{e^{\frac{1}{2} x+\frac{1}{2} x^{4}}}{\sqrt{1-x}}
$$

wherefore we have

$$
\phi(m, 0)=1.2 \ldots m \text { coefft. } x^{m} \text { in } \frac{e^{\frac{1}{2} x+\frac{1}{2} x^{2}}}{\sqrt{1-x}}
$$


Developing as far as $x^{6}$, the numerical process is

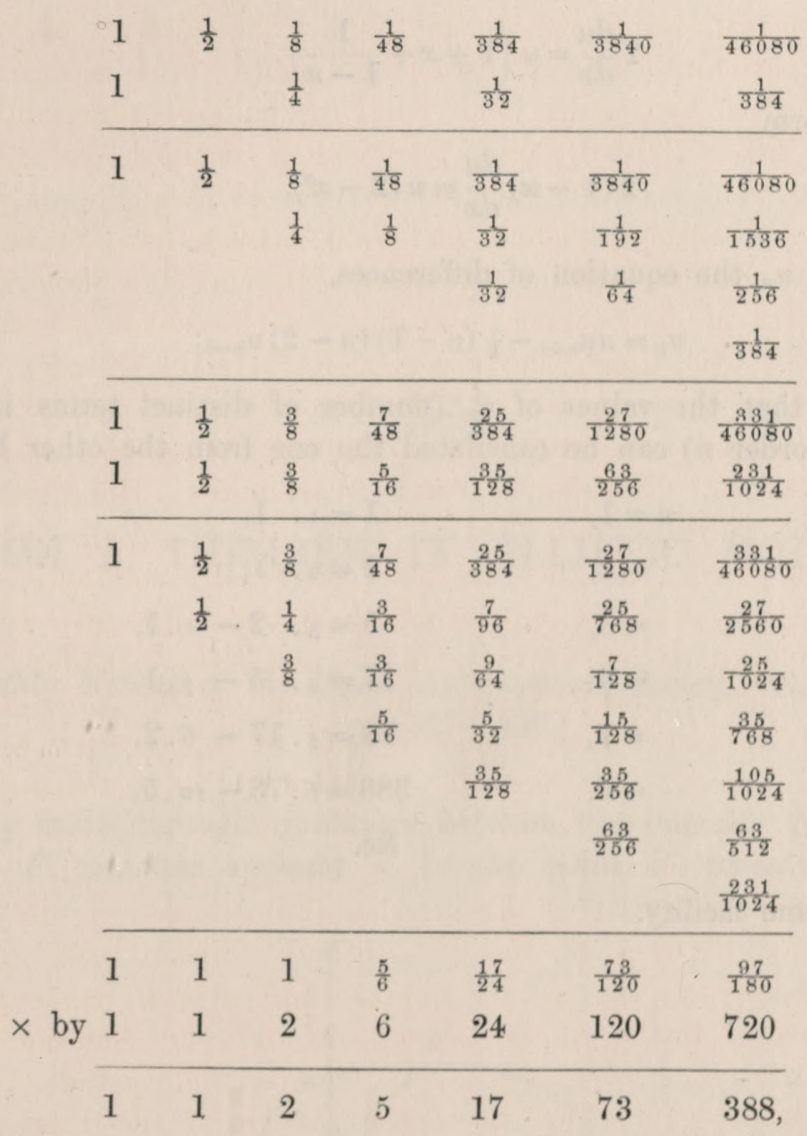

agreeing with the former values.

The expression of $\phi(m, 0)$ once found, it is easy thence to obtain

$$
\begin{aligned}
& \phi(m, 1)=1.2 \ldots m \text { coefft. } x^{m} \text { in } \frac{e^{\frac{1}{3 x+3 x^{2}}}}{(1-x)^{\frac{3}{2}}} \\
& \phi(m, 2)=1.2 \ldots m \text { coefft. } x^{m} \text { in } \frac{2 e^{1 x+\frac{3}{2} x^{2}}}{(1-x)^{\frac{5}{2}}} \\
& \phi(m, 3)=1.2 \ldots m \text { coefft. } x^{m} \text { in } \frac{2.3 e^{\frac{1}{3} x+\frac{1}{3} x^{2}}}{(1-x)^{\frac{7}{2}}}
\end{aligned}
$$

and so on, the law being obvious.

[Addition, p. 335.] The generating function

$$
u,=1+u_{1} x+\ldots+u_{n} \frac{x^{n}}{1.2 \ldots n}+\ldots,=\frac{e^{\frac{1}{3 x+13 x^{2}}}}{\sqrt{1-x}},
$$


was obtained as the solution of the differential equation

$$
2 \frac{d u}{d x}=u\left(1+x+\frac{1}{1-x}\right)
$$

Writing this in the form

$$
2(1-x) \frac{d u}{d x}=u\left(2-x^{2}\right)
$$

we at once obtain for $u_{n}$ the equation of differences,

$$
u_{n}=n u_{n-1}-\frac{1}{2}(n-1)(n-2) u_{n-3} ;
$$

and it thus appears that the values of $u_{n}$ (number of distinct terms in a symmetrical determination of the order $n$ ) can be calculated the one from the other by the process

$$
\begin{array}{rlrl}
n=1, & 1=\text { r. } 1, \\
=2, & 2=2.1, \\
=3, & 5=3 \cdot 2-1.1, \\
=4, & 17=4 \cdot 5-3.1, \\
=5, & 73=5 \cdot 17-6.2, \\
=6, & 388=6.73-10.5, \\
& & \text { \&c. }
\end{array}
$$

which is one of extreme facility. 